\title{
Multi-Radio Perspectives for Massive MTC Localization: Energy Consumption and Utility
}

\author{
Konstantin Mikhaylov*†\|, Maria A. Lema ${ }^{\ddagger}$, Sergey Andreev ${ }^{\S \ddagger}$, Rohit Gupta ${ }^{\uparrow}$, Olga Galinina ${ }^{\S}$, \\ Giuseppe Destino ${ }^{\ddagger *}$, Toktam Mahmoodi ${ }^{\ddagger}$, Mischa Dohler ${ }^{\ddagger}$, Yevgeni Koucheryavy ${ }^{\S}$, and Mikko Valkama ${ }^{\S}$ \\ *University of Oulu, Oulu, Finland \\ ${ }^{\dagger}$ Brno University of Technology, Brno, Czech Republic \\ $\ddagger$ King’s College London, London, United Kingdom \\ $\S$ Tampere University, Tampere, Finland \\ ףActility S. A., Paris, France \\ "Email: konstantin.mikhaylov@oulu.fi
}

\begin{abstract}
The value of the location data for the Internet of Things (IoT) devices is undisputed, and today's radio technologies provide various means to obtain these data. In this paper, we shed light on the utility of the four illustrative radio-based localization techniques, including satellite navigation and the use of BLE, LoRaWAN, and Cat-NB1 communication technologies. First, we instrument a prototype device comprising all these solutions, thus confirming the feasibility of multi-radioenabled IoT devices supporting localization. Then, we employ this prototype to empirically assess the energy utility of different localization approaches. Our results demonstrate that in terms of energy consumption, the difference between these techniques approaches five orders of magnitude. By conducting a follow-up survey of the relevant research papers, we assess other important performance metrics, such as the operating environment and the required infrastructure, as well as their effect on the localization accuracy. By combining these results, we show that multi-radioenabled localization solutions are more flexible than those based on a single localization solution and provide space for further optimizations, especially with regards to energy consumption. Development of such devices is an crucial step toward enabling "anytime, anywhere" localization for IoT devices.
\end{abstract}

Index Terms-IoT, localization, positioning, multi-RAT, MTC, NB-IoT, BLE, GNSS

\section{INTRODUCTION}

Decisive growth in the number of connected autonomous machines deployed all around us marks the inception of the Internet of Things (IoT) [1] era. In the years to come, myriads of new devices will spread to enable new exciting use cases and applications. Aside from the "anytime, anywhere" connectivity requirement to be satisfied by both existing and emerging communication technologies, another challenge of extreme importance for the IoT applications is enabling efficient localization of machine-type devices.

Indeed, for some applications (e.g., pet, goods, or belongings tracking), identifying the device location and delivering the respective information is an objective by itself. For others (e.g., swarms of sensing drones), location data is crucial to establish the context of the measurements. Moreover, the location information plays a pivotal role in optimizing and supporting the sustainability of the IoT ecosystem as a whole, thus enabling devices to determine their mutual position and that of the surrounding infrastructure. The latter is valuable not only for device and network maintenance but also reveals important context information to strengthen security and provides grounds for optimizing connectivity at various layers of the IoT ecosystem.

The contemporary technology base offers a sheer diversity of localization solutions based on different principles. Among these are the use of artificially-generated signals (e.g., acoustic, ultrasonic, radio frequency (RF), light, and infrared), measurement of environment factors (strength of an electromagnetic field, level of light or noise, etc.), inertial positioning with respect to a known starting point, as well as the use of machine vision. Among all of these, the RF signal based systems [2] are presently the most widely employed due to their low cost, substantial coverage, decent accuracy, and time performance.

The diversity of the machine-type communication (MTC) landscape available today [3] provides multiple opportunities beyond using the conventional global navigation satellite system (GNSS). The Low-Power Wide-Area Network (LPWAN) based solutions can become the basis for implementing network-centric positioning (NCP) [4] by employing triangulation. An infrastructure of beacon nodes that broadcast $\mathrm{WiFi}, \mathrm{BLE}$, or ZigBee signals provides the means for devicecentric positioning (DCP), while cellular IoT systems are able to support both NCP and DCP. Finally, centimeter-accuracy localization within a limited area can be accomplished by utilizing the Ultra Wideband (UWB) radio signals.

Naturally, the alternative approaches have their strengths and weaknesses. Despite being efficient in open-space outdoor scenarios, GNSS devices may experience difficulties receiving satellite signals indoors. Other systems require an appropriate infrastructure in place, which may not always be available. These controversial trends lead us to a conclusion that hardly there is a single localization technology today, which is capable of enabling localization "anytime, anywhere." What is more, recently there emerged concerns related to increased energy consumption levels of the telecommunication systems and their negative environmental impact [5], thus calling for minimizing the energy consumption both at the network infrastructure and the device sides.

Motivated by these two facts, this paper studies the feasi- 
TABLE I

CHARACTERISTICS OF SELECTED WIRELESS LOCALIZATION AND COMMUNICATION TECHNOLOGIES

\begin{tabular}{|c|c|c|c|c|}
\hline Technology: & BLE & LoRaWAN & Cat-NB1 & GNSS \\
\hline Frequency band & $2.4 \mathrm{GHz}$ ISM & $868 \mathrm{MHz}$ ISM & $700-2100 \mathrm{MHz}$ lic. & up to $30 \mathrm{MHz}$ \\
Bandwidth & 1 or $2 \mathrm{MHz}$ & $125-500 \mathrm{kHz}$ & $200 \mathrm{kHz}$ & public \\
Infrastructure & private & private/operator & operator & long \\
Range & short & long & various \\
Modulation & GFSK & LoRa & DPS and/or QPSK & DCP \\
Architecture & DCP/NCP & NCP & uplink/downlink & none \\
Data traffic & uplink/downlink & uplink/downlink & \\
\hline
\end{tabular}

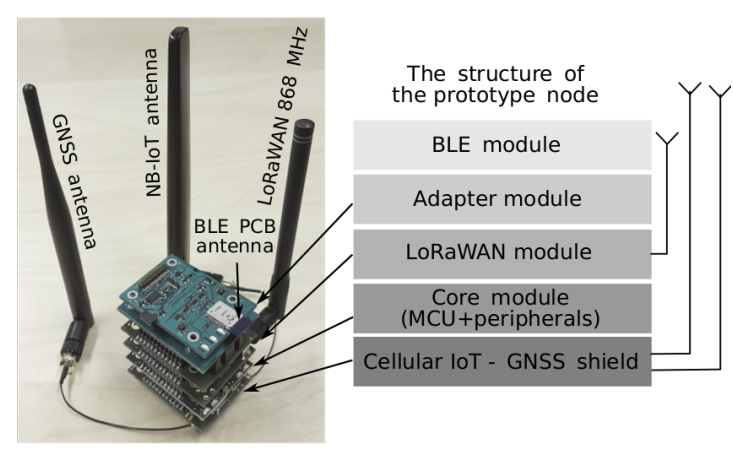

(a)
Supervision and control thread (periodic wakeup, activation of low-level thread, LPM management)

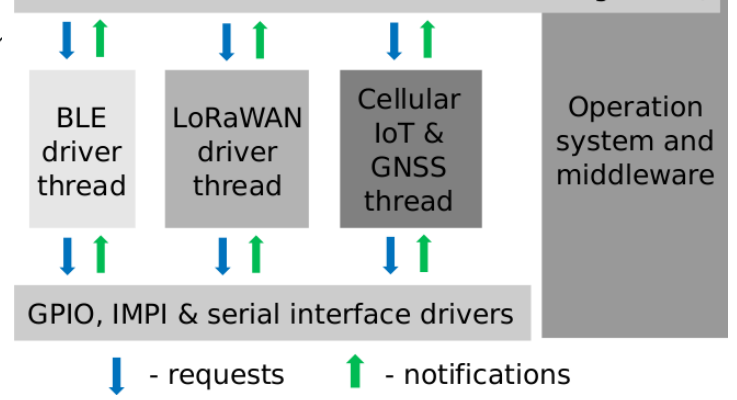

(b)

Fig. 1. Photo and architecture of the prototyped device (a) and structure of its firmware (b)

bility and benefits of a multi-radio-enabled IoT device that is capable of utilizing several localization technologies. The IoT devices capable of communicating over multiple radio technologies have recently been introduced, e.g., in [6]. Therefore, in this paper, we focus on a resource-limited localization and, specifically, on its energy aspect. The demonstration of feasibility of the said IoT device and assessment of its energy profile form a major technical contribution of this study. We also assess other key performance metrics, such as operating environment and required infrastructure as well as their effect on the localization accuracy by surveying the relevant research papers. These results together confirm feasibility and benefits of multi-RAT-enabled localization in the context of IoT, thus justifying and motivating further studies of such systems.

\section{Design and Evaluation of a Multi-RAT LOCALIZABLE IOT DEVICE}

\section{A. Technology selection}

For our implementation, we intentionally select illustrative radio technologies representing different groups of MTC connectivity and localization options. Their main characteristics are summarized in Table I. Today, GNSS-based localization is the most widely utilized solution for outdoor scenarios. In our device, we support multiple global and local NSS, namely, GPS, BeiDou, Galileo, GLONASS, and QZSS.

Bluetooth Low Energy (BLE) is an energy efficient shortrange technology operating in the only globally uniform license-free industrial, scientific, and medical (ISM) band of 2.4 GHz. Nowadays, Bluetooth is the second most widely used IoT communication technology after $\mathrm{WiFi}^{1}$. The recently

\footnotetext{
${ }^{1}$ Business Insider Intelligence, available: http://www.businessinsider.com/inmarsat-acility-lpwan-iot-2017-2
}

released 5.0 version of the specification has introduced three new physical layers and link-layer features, which enable improved throughput and communication range.

LoRaWAN is selected as the most widely used LPWAN technology operating in license-free sub-GHz ISM band ${ }^{2}$. Aside from this, LoRaWAN is one of the few LPWAN technologies, which supports the deployment of both continentwide public and local private networks. The latter is especially important for delivering connectivity to remote and/or isolated locations, such as warehouses, hospitals, or campuses.

Long Term Evolution (LTE) Cat-NB1 represents the way how the need for enabling cost- and energy-efficient IoT communication is addressed by the Third Generation Partnership Project (3GPP). Unlike the two above solutions, this one is primarily intended to operate in the licensed bands of the traditional telecom operators. This potentially increases the operating expenditures per device but provides more reliable delivery of their data.

\section{B. Architecture and design}

A proof-of-concept multi-RAT-enabled localizable device has been prototyped by using our in-house modular IoT platform [7]. A photo of the designed prototype along with its structural diagram is offered in Fig. 1. The photos of the prototype, its hardware architecture, and software composition are depicted in Fig. 1.

The prototype comprises two major components. The former one is the core module composed of the STM32F217 32-bit microcontroller and a DC-DC regulatory circuitry. To the top and the bottom of the core module, we stack three

$$
{ }^{2} \text { A. Weissberger, ComSoc blog, available: }
$$
http://techblog.comsoc.org/2017/10/25/lora-wan-and-sigfox-lead-lpwansinteroperability-via-compression/ 


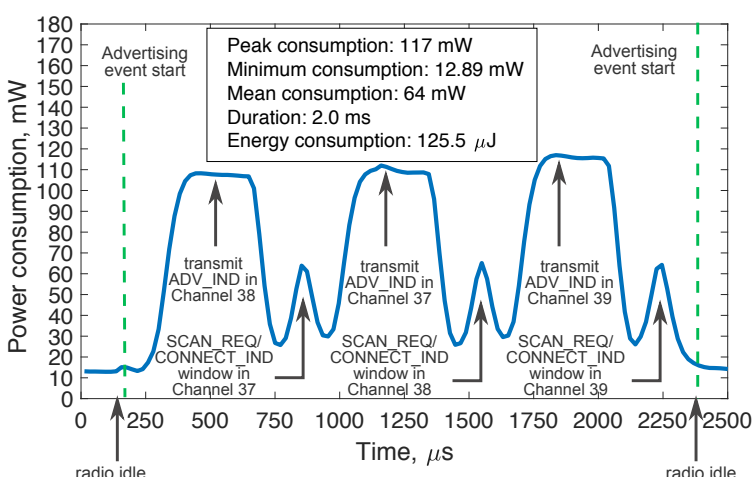

(a)

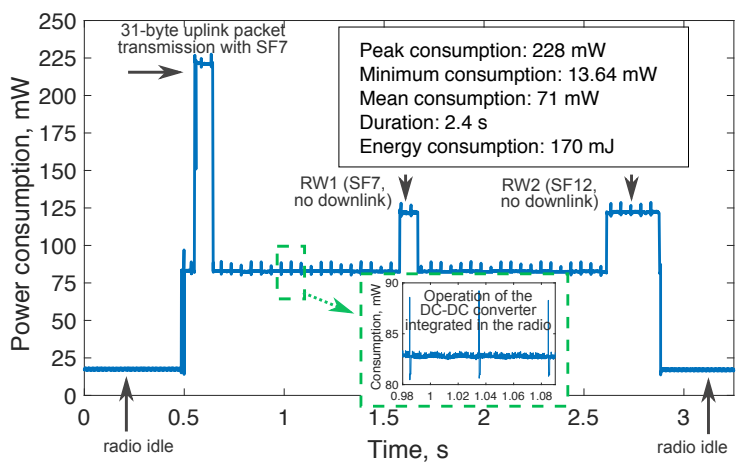

(c)

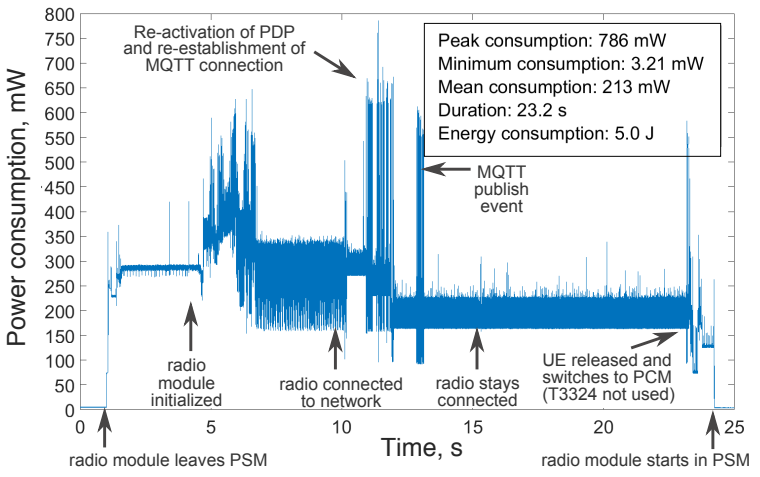

(e)

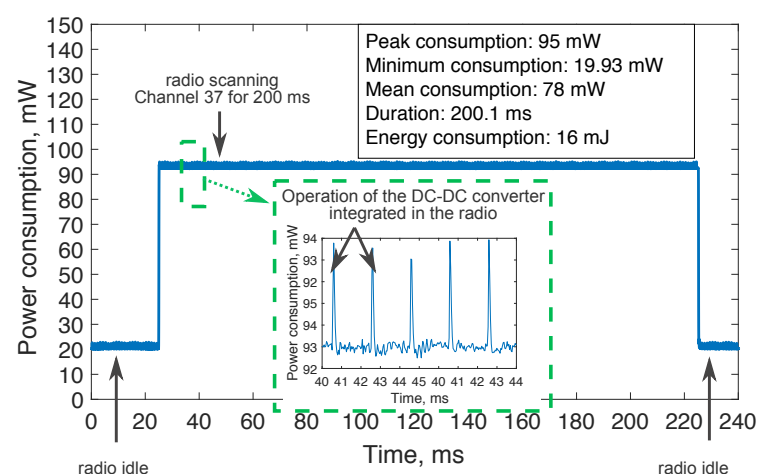

(b)

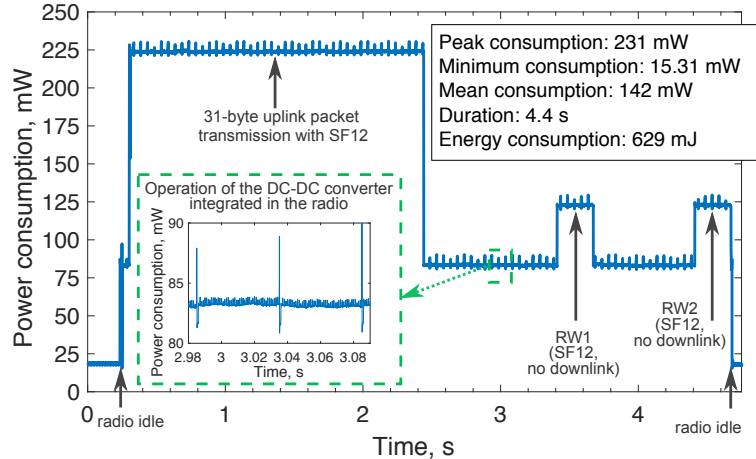

(d)

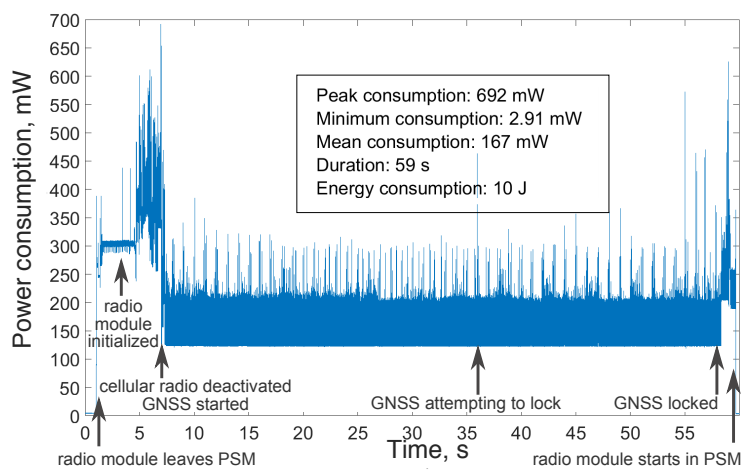

(f)

Fig. 2. Power consumption profile of the device using different technologies: (a) BLE for NCP, (b) BLE for DCP, (c) LoRaWAN SF7 for NCP, (d) LoRaWAN SF12 for NCP, (e) Cat-NB1 for NCP, (f) GNSS

radio modules featuring commercial BLE, LoRaWAN, and Cat-NB1 transceivers, as well as a GNSS receiver integrated within a single module. The in-house developed radio modules are built around Microchip RN4020 BLE module, Microchip RN2483 LoRaWAN module (firmware version 1.0.3), Quectel BG96 Cat-NB1/Cat-M1+GNSS module (firmware version 02A07M1G). An adapter board has been added before the BLE module to convert the physical interfaces between the two versions of the modular platform [8].

The firmware is built around the industry-grade multithreading FreeRTOS operating system, extended with the necessary drivers. A separate thread has been implemented to control each radio transceiver and the GNSS receiver. A control thread has been developed to manage all of the active radio technologies. This architecture provides the necessary flexibility for the parallel use of different RATs and allows for easy switching between the various technologies in our measurements. Within each of the threads, the respective radio transceiver is configured and then periodically provided with a beacon packet to send. Implying that the device is in the coverage range of several base stations or anchor nodes, the latter may use triangulation for NCP. An alternative firmware has been implemented for BLE: it switches the radio to the scanning mode, thus enabling reception of infrastructure broadcasts to facilitate DCP. Within the GNSS thread, the microcontroller periodically starts the GNSS receiver, waits for it to lock and report the coordinates, and switches the receiver to a low power mode. 


\section{Testbed setup and evaluation methodology}

During power consumption measurements, the test device has been restricted to only one technology at a time, and the power consumption profile of the device has been recorded by using Agilent N6705B power analyzer. The analyzer is configured to provide $3.5 \mathrm{~V}$ stable supply to the main power rail of the device. For BLE, the sampling rate is 50 kilosamples/s over 30 second period, for all the other cases -10 kilosamples/s over 10-minute intervals, during which multiple localization events occur. The measurements were conducted in an office environment. The transmit power for BLE and LoRaWAN was fixed at $0 \mathrm{dBm}$ and $14 \mathrm{dBm}$, correspondingly. While measuring Cat-NB1, the test device has been connected to the network of one of the commercial Finnish telecom operators. For each of the technologies, multiple experiments have been conducted. All recorded current consumption profiles have been further post-processed (e.g., the $17 \mathrm{~mA}$ static consumption of on-board peripherals has been compensated for) by using MATLAB to estimate the duration and the amount of energy needed for a single localization event.

\section{RESULTS AND DISCUSSION}

\section{A. Energy consumption}

The selected results of our measurements are presented in Fig. 2 and Fig. 3. The use of BLE results in the lowest energy consumption - for NCP, a single localization event requires less than two hundred microJoules, for DCP - several dozens of milliJoules are consumed. This is due to the high on-air data rate and the limited transmit power of BLE. Note that for DCP, the consumption is dependent on the beacon advertising interval (i.e., advInterval parameter), which may take values between $20 \mathrm{~ms}$ and 2.78 hours $^{3}$.

The measured consumption of a class A LoRaWAN transceiver for NCP ranged from $170 \mathrm{~mJ}$ to $294 \mathrm{~mJ}$, for the minimum and the maximum spreading factor (SF) possible in Europe. In the profile, one may observe that the transceiver first transmits its data and then provides up to two receive windows for potential downlink communication. Higher spreading factors, which result in longer on-air time, enable achieving a broader range of communication. The SF employed by a device can be either fixed or dynamically adjusted depending on the channel conditions. Note that the LoRaWAN architecture (refer to Fig. 1 in [9]) is already well-suited for implementing NCP. Each LoRaWAN gateway $(\mathrm{GW})$ in a network transfers all the received uplink packets to a Network Server (NS), which may exploit triangulation to locate the devices.

Since neither the Cat-NB1 transceivers nor the base stations of today support localization, we demonstrate a first-order approximation (for the worst-case scenario) by measuring the consumption of a complete uplink communication event. Transmission of a single uplink frame over Cat-NB1 requires

\footnotetext{
${ }^{3}$ In our measurements, we assume it equal to $90 \mathrm{~ms}$ with up to ten seconds extra due to the random component. Therefore, the scanning time is set to $200 \mathrm{~ms}$.
}

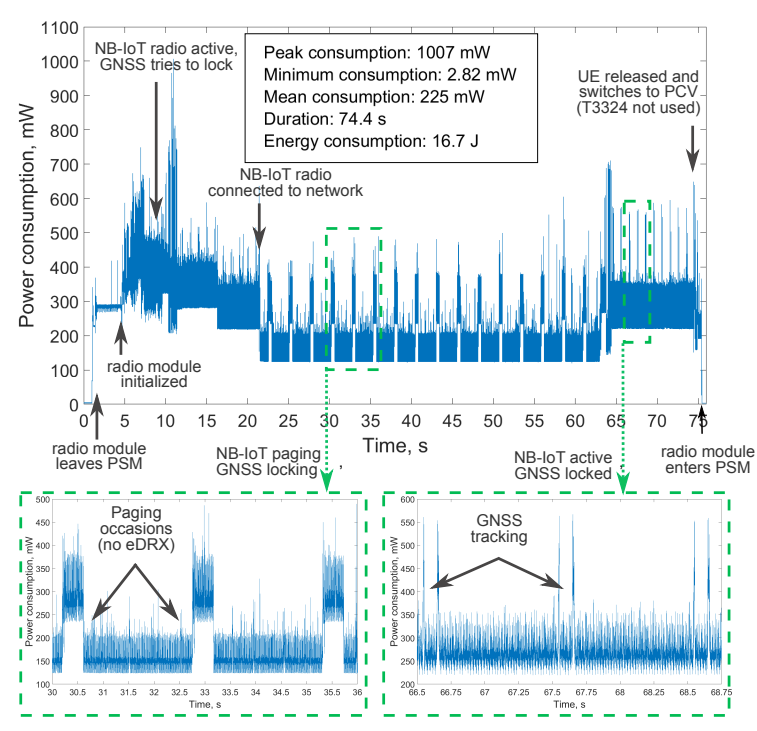

Fig. 3. Power consumption profile of device localization by using GNSS and reporting over LTE Cat-NB1

as much as $5 \mathrm{~J}$ of energy. After leaving the power saving mode (PSM), the transceiver re-establishes its connection to the network, activates the packet data protocol contexts, and sends data using the Message Queuing Telemetry Transport (MQTT) protocol. Once the data are sent, the NB-IoT transceiver maintains the connection unless the network releases it and then switches back to PSM (timer T3314 is skipped).

Note that the amount of consumed energy is also affected by the channel conditions and network configurations (e.g., set value for the Radio Resource Connection (RRC) release timer). In our experiments, we register the duration of a single uplink communication event of 20 to 60 seconds. Furthermore, unlike the mentioned BLE and LoRaWAN transceivers, which use the "idle" mode between the transmissions, the NBIoT transceiver enters the PSM, which results in a lower consumption in-between the localization events (minimum power consumption in the charts). Given that the low power modes are design-specific, we do not consider them to be illustrative for the discussed technologies.

Finally, the highest amount of energy - of over 10 Joules is consumed when using the GNSS receiver. In the illustrated case, the receiver requires almost one minute to acquire the signals from the expected number of satellites and determine its position. Throughout our experiments, we also observe a significant deviation of the GNSS receiver locking times, which ranged from several dozens of seconds to more than ten minutes depending on the time of the day and location of the receiver. The high energy consumption at the initial phase is caused by the operation of the Cat-NB1/Cat-M1 transceiver, with which the GNSS receiver employed in our experiments is integrated, and which automatically starts in the active mode after leaving PSM and needs to be stopped by the microcontroller.

Finally, Fig. 3 illustrates the consumption of a device performing GNSS localization and reporting its coordinates over 
TABLE II

SELECTED KEY PERFORMANCE INDICATORS OF TARGETED LOCALIZATION TECHNOLOGIES

\begin{tabular}{|c|c|c|c|c|c|c|}
\hline Technology & Ref. & Methodology & Principle & Environment & Test area & Accuracy \\
\hline BLE & {$[10]$} & experiment & $\mathrm{DCP}^{\mathrm{a}}, \mathrm{RSS}$ & indoor (museum) & $5 \times 2 \mathrm{~m}, 6$ beacons & below $1 \mathrm{~m}$ CDF $90 \%$ \\
\hline BLE & [11] & experiment & $\mathrm{DCP}^{\mathrm{a}}$, RSS fingerpr. & indoor (conf.room) & $17.5 \times 9.6 \mathrm{~m}, 10$ beacons & $2 \mathrm{~m} \mathrm{CDF} 90 \%$ \\
\hline BLE & [12] & experiment & $\mathrm{NCP}^{\mathrm{b}}$, dir. of departure & indoor & $6 \times 7 \mathrm{~m}, 1$ beacon & $1 \mathrm{~m} \max$ \\
\hline BLE & [13] & experiment & $\mathrm{DCP}^{\mathrm{a}}, \mathrm{RSS}$ & indoor (conf. room) & $17.5 \times 9.6 \mathrm{~m}, 10$ beacons & below 2 m CDF $90 \%$ \\
\hline BLE & [11] & experiment & $\mathrm{DSB}^{\mathrm{a}}$ & indoor & $110 \mathrm{~m}$ square, 3 beacons & $7 \mathrm{~m} \max$ \\
\hline BLE & [14] & experiment & $\mathrm{DCP}^{\mathrm{a}}, \mathrm{RSS}$ & indoor (conf. room) & $5.6 \times 5.9 \mathrm{~m}, 3$ beacons & $1.1 \mathrm{~m} \mathrm{max}$ \\
\hline LoRaWAN & [14] & experiment & $\mathrm{DCP}^{\mathrm{a}}, \mathrm{RSS}$ & indoor (conf. room) & $5.6 \times 5.9 \mathrm{~m}, 3$ beacons ${ }^{\mathrm{c}}$ & $2.71 \mathrm{~m} \max$ \\
\hline LoRaWAN & [15] & experiment & NCP, TDoA & outdoor & $3 \times 3 \mathrm{~km}, 4$ GWs & $100 \mathrm{~m}$ mean \\
\hline LoRaWAN & [16] & experiment & $\mathrm{NCP}^{\mathrm{b}}, \mathrm{TDoA}$ & outdoor & $3 \times 3 \mathrm{~km}, 3 \mathrm{GWs}$ & $550 \mathrm{~m}$ with CDF $90 \%$ \\
\hline LoRaWAN & [17] & experiment & $\mathrm{DCP}^{\mathrm{a}}, \mathrm{RSS}$ & outdoor & $100 \mathrm{~m}$ circle, 8 beacons ${ }^{\mathrm{a}}$ & $7.5 \mathrm{~m}$ mean \\
\hline Cat-NB1 & [18] & experiment & $\mathrm{DCP}^{\mathrm{a}}$, channel fingerpr. & indoor (research lab) & $12 \times 15 \mathrm{~m}$ & $2 \mathrm{~m}$ with CDF $89 \%$ \\
\hline Cat-NB1 & [19] & simulation & $\mathrm{DCP}^{\mathrm{a}}, \mathrm{TDoA}$ & indoor & $\mathrm{n} / \mathrm{a}^{\mathrm{d}}, 4$ sites $700 \mathrm{~m}$ apart & $160 \mathrm{~m}$ with CDF $80 \%$ \\
\hline Cat-NB1 & [19] & simulation & $\mathrm{DCP}^{\mathrm{a}}$, TDoA & outdoor & $\mathrm{n} / \mathrm{a}^{\mathrm{d}}, 4$ sites $700 \mathrm{~m}$ apart & 150 m with CDF $90 \%$ \\
\hline Cat-NB1 & [20] & simulation & $\mathrm{DCP}^{\mathrm{a}}, \mathrm{TDoA}$ & outdoor & $4 \times 4 \mathrm{~km}, 6$ sites & 140 m with CDF $80 \%$ \\
\hline GNSS & [21] & experiment & $\mathrm{DCP}^{\mathrm{a}}, \mathrm{ToA}$ & outdoor (open sky) & $\mathrm{n} / \mathrm{a}^{\mathrm{d}}$ & 3 m with CDF $90 \%$ \\
\hline GNSS & [22] & experiment & $\mathrm{DCP}^{\mathrm{a}}, \mathrm{ToA}$ & outdoor (urban) & $\mathrm{n} / \mathrm{a}^{\mathrm{d}}$ & $15 \mathrm{~m} \max$ \\
\hline GNSS & [21] & experiment & $\mathrm{DCP}^{\mathrm{a}}, \mathrm{ToA}$ & outdoor (open sky) & $\mathrm{n} / \mathrm{a}^{\mathrm{d}}$ & 12 m with CDF $89 \%$ \\
\hline GNSS & [23] & experiment & $\mathrm{DCP}^{\mathrm{a}}$, ToA & outdoor (urban) & $\mathrm{n} / \mathrm{a}^{\mathrm{d}}$ & $7 \mathrm{~m} \mathrm{RMS}$ \\
\hline GNSS & [24] & experiment & $\mathrm{DCP}^{\mathrm{a}}$, ToA & indoor & $\mathrm{n} / \mathrm{a}^{\mathrm{d}}$ & $75 \mathrm{~m}$ RMS \\
\hline
\end{tabular}

Cat-NB1. The presented results conclusively show that the localization mechanisms based on various radio technologies differ significantly with respect to their energy consumption. As one may conclude from the above examples, this difference may easily reach up to five orders of magnitude.

\section{B. Other critical performance metrics}

To meticulously assess the critical performance metrics, which cannot be determined from our experimental data but remain essential for understanding the utility of different localization techniques, we build our further discussion on the results of the previous studies. The essential details related to the adopted methodology, experimental setup, and results are summarized in Table II.

The analysis of these results shows that BLE is primarily utilized for localizing the devices indoor at the dozen-meter distances. The localization is performed by using RSS in either NCP or DCP manner, but the latter is more common; the expected accuracy is approximately one meter. The LoRaWAN has been experimentally tested both indoors and outdoors, providing few-meter accuracy for dense indoor infrastructures and hundred-meter accuracy for sparse urban outdoor deployments. The localization may be performed by using either RSS or TDoA, and is typically implemented as NCP. Similar to LoRaWAN, Cat-NB1 permits for localization based on RSS or TDoA, with the latter prevalent. Unlike LoRaWAN, Cat-NB1 is expected to be used primarily in DCP. The accuracy of Cat-NB1 will likely be on the same order as that of LoRaWAN. However, due to the novelty of Cat-NB1 technology, few results of the practical experiments have been reported. Finally, GNSS provides few-meter accuracy under the open sky and dozen- to hundred-meters accuracy in more challenging conditions.

\section{MAIN CONCLUSIONS}

Although the diversity of localization solutions for IoT devices remains a particular challenge for the network de- signers of today, it also brings new attractive opportunities. In this paper, we considered the practicalities of the state-ofthe-art and prospective localization approaches, one of which includes the use of GNSS, and the rest are based on three communication technologies - BLE, LoRaWAN, and CatNB1. We started by prototyping a real-world device capable of utilizing all four radio technologies, thus demonstrating the feasibility of such systems.

Then, we tested our developed prototype to gain insights into the energy consumption of the considered localization approaches individually. Our results show that in terms of energy consumption, the difference between the localization techniques might grow up to five orders of magnitude. To obtain a better understanding of the important performance metrics, such as the operating environment and infrastructure as well as their effect on the localization accuracy, we conduct an extensive survey of the relevant research papers. The results of these studies demonstrate that aside from the energy consumption, various localization methods differ with respect to their performance and operating environment. The key takeaways of these activities are summarized in Table III.

Specifically, short-range radio technologies (as, for example, BLE) are characterized by low energy consumption and fast operation but require relatively dense infrastructure deployments. These aspects make short-range systems most suitable for indoor locations where positioning of multiple objects is known to be required (e.g., warehouses or shopping malls), especially given their better scalability due to shorter on-air time. The LPWAN-based solutions enable positioning both indoors and outdoors but imply higher energy costs. The LoRaWAN operating in license-free bands supports well the private networks, where the density and location of gateways may be optimized for tracking, which makes LoRaWAN technology well-suited for network-centric localization.

The Cat-NB1 and cellular IoT technologies may be somewhat less flexible with respect to deploying base stations, 
TABLE III

SUMMARY OF KEY PERFORMANCE INDICATORS FOR TARGETED LOCALIZATION TECHNOLOGIES

\begin{tabular}{|c|c|c|c|c|}
\hline Technology: & BLE & LoRaWAN & Cat-NB1 & GNSS \\
\hline Localization type & device-centric & network-centric & device-centric & device-centric \\
\hline Localization method & RSS & AoA+RSS/TDoA & TDoA & ToA \\
\hline Typical environment & indoor & indoor/outdoor & indoor/outdoor & outdoor \\
\hline Accuracy & units of meters & dozens - hundreds meters & dozens - hundreds meters & meters $^{\mathrm{a}}$ \\
\hline Infrastructure density & high & high/low & low & $\mathrm{n} / \mathrm{a}$ \\
\hline Localization delay & $\mathrm{ms}$ & $\mathrm{ms}-\mathrm{s}$ & $\mathrm{ms}-\min$ & dozens $\mathrm{s}$ - dozens min \\
\hline Typical consumption (per event) & $\mathrm{mJ}$ & dozens $\mathrm{mJ}-\mathrm{J}$ & hundreds $\mathrm{mJ}^{\mathrm{b}}$ - dozens $\mathrm{J}$ & dozens - hundreds $\mathbf{J}$ \\
\hline Device cost & 1 USD & $2-3$ USD & 5 USD & $5-10$ USD \\
\hline Infra cost per anchor & dozens USD & hundreds USD & $\mathrm{n} / \mathrm{a}$ & $\mathrm{n} / \mathrm{a}$ \\
\hline Subscription cost (per device) & none $\mathrm{e}^{\mathrm{c}}$ & none $^{\mathrm{C}}-1 \mathrm{USD} / \mathrm{month}$ & $1 \mathrm{USD} / \mathrm{month}$ & none \\
\hline
\end{tabular}

but they hold potential opportunities for device-centric localization. Finally, while a GNSS receiver features substantial time and consumed energy to lock (especially in a coldstart scenario), it requires neither additional costs nor any private infrastructure to be deployed. The multi-radio-enabled device prototyped and described in this paper can utilize any of these technologies or their desired combination, thus benefiting from this diversity. Understanding the limitations of the single-technology approach taken in this work, we plan to further study the utility of simultaneous multi-RAT localization, which is enabled by the developed platform.

In summary, the results provided in this paper could be useful for practitioners seeking a balanced combination of radio technologies to integrate into their products. Moreover, our study shows that enabling multiple localization technologies allows devices to flexibly optimize their operation with respect to the consumed energy and, therefore, substantially reduce the energy expenditures as well as, potentially, improve the reliability. Consequently, the development of optimization algorithms for multi-RAT-enabled localization may represent a new attractive opportunity with high pay-offs.

Another interesting question, which we have not touched in this work, is related to the simultaneous utilization of multiple radio technologies. Can this approach enable increased localization accuracy and, if yes, to which extent? How to combine and post-process the data received from multiple technologies? Does this make sense from the energy consumption perspective? These questions remain to be investigated.

\section{ACKNOWLEDGMENT}

This work is supported by the Academy of Finland 6Genesis Flagship (grant 318927) as well as projects PRISMA, WiFiUS, and 5G-FORCE. The work of K. Mikhaylov was also supported by the mobility project MeMoV, No. CZ.02.2.69/0.0/0.0/16_027/00083710, of Czech Ministry of Education, Youth, and Sport, funded by European Social Fund and LPWAN-evolution activity funded by the University of Oulu. The work of O. Galinina is supported by a personal Jorma Ollila grant from the Nokia Foundation, by the Finnish Cultural Foundation, and by a Postdoctoral Researcher grant from the Academy of Finland.

\section{REFERENCES}

[1] A. Zanella et al., "Internet of Things for Smart Cities," IEEE IoT J., vol. 1, pp. 22-32, 2014.
[2] H. Liu et al.,"Survey of Wireless Indoor Positioning Techniques and Systems," IEEE Trans. Syst., Man, Cyber., vol. 37, pp. 1067-1080, 2007.

[3] S. Andreev et al., "Understanding the IoT connectivity landscape: a contemporary M2M radio technology roadmap," IEEE Commun. Mag., vol. 53, pp. 32-40, Sept. 2015.

[4] G. Destino, "Positioning in wireless networks: non-cooperative and cooperative algorithms," Ph. D. thesis, Univesity of Oulu, 2012.

[5] C. Zhu et al., "Green Internet of Things for Smart World," IEEE Access, vol. 3, pp. 2151-2162, 2015.

[6] K. Mikhaylov et al., "Energy Efficiency of Multi-Radio Massive Machine-Type Communication (MR-MMTC): Applications, Challenges, and Solutions," IEEE Commun. Mag., vol. 57, pp. 100-106, Apr. 2019.

[7] K. Mikhaylov and J. Petajajarvi, "Design and Implementation of the Plug\&Play enabled Flexible Modular Wireless Sensor and Actuator Network Platform," Asian J. Control, vol. 19, pp. 1-21, Sept. 2017.

[8] K. Mikhaylov, "Plug and play reconfigurable solutions for heterogeneous IoT," Ph. D. thesis, Univesity of Oulu, 2018.

[9] K. Mikhaylov, J. Petajajarvi, and J. Janhunen, “On LoRaWAN Scalability: Empirical Evaluation of Technology Succeptability to In Network Interference", in Proc. EuCNC'2017, June 12-15, pp. 1-6.

[10] A. R. Jimenez and F. Seco, "Finding objects using UWB or BLE localization technology: A museum-like use case", in Proc. IPIN'2017, 2017, pp. 1-8.

[11] C. Xiao et al., "3-D BLE Indoor Localization Based on Denoising Autoencoder," IEEE Access, vol. 5, pp. 12751-12760, 2017.

[12] K. Kikuchi et al., "Feasible calibration of DOD-based BLE beacon for indoor localization application,” in Proc. ISAP'2017, 2017, pp. 1-2.

[13] A. De Blas and D. Lopez-de-Ipina, "Improving trilateration for indoors localization using BLE beacons," in Proc. SpliTech'2017, 2017, pp. 1-6.

[14] S. Sadowski and P. Spachos, "RSSI-Based Indoor Localization with the Internet of Things," IEEE Access, vol. 6, pp. 30149-30161, 2018.

[15] B. C. Fargas and M. N. Petersen, "GPS-free geolocation using LoRa in low-power WANs," in Proc. GIoTS'2017, 2017, pp. 1-6.

[16] N. Podevijn et al., "TDoA-Based Outdoor Positioning with Tracking Algorithm in a Public LoRa Network," Wireless Commun. Mobile Comput., vol. 2018, paper 1864209, 2018.

[17] K. H. Lam et al., "New RSSI-Based LoRa Localization Algorithms for Very Noisy Outdoor Environment," in Proc. COMPSAC'2018, 2018, pp. 794-799.

[18] Q. Song et al., "CSI Amplitude Fingerprinting-Based NB-IoT Indoor Localization,” IEEE IoT J., vol. 5, pp. 1494-1504, 2018.

[19] X. Lin et al., "Positioning for the Internet of Things: A 3GPP Perspective," IEEE Commun. Mag., vol. 55, pp. 179-185, 2017.

[20] S. Hu et al., "Improving the Performance of OTDOA Based Positioning in NB-IoT Systems," in Proc. GLOBECOM'2017, 2017, pp. 1-7.

[21] K. A. B. Ahmad et al., "Characterization of GNSS Receiver Position Errors for User Integrity Monitoring in Urban Environments," in Proc. ENC'2014, 2014, pp. 1-15.

[22] E. Rademakers et al., "Obtaining real-time sub-meter accuracy using a low cost GNSS device," in Proc. ENC'2016, 2016, pp. 1-8.

[23] P. D. Groves and M. Adjrad, "Likelihood-based GNSS positioning using LOS/NLOS predictions from 3D mapping and pseudoranges," GPS Solutions, vol. 21, pp. 1805-1816, 2017.

[24] G. Lachapelle, "GNSS Indoor Location Technologies," in Proc. GNSS'2004, 2004, pp. 1-15. 